\title{
VALORACIÓN NUTRICIONAL DE GRANOS DE CAFÉ ROBUSTA (Coffea canephora) DE DIFERENTES ORÍGENES PROCESADOS EN MÉXICO
}

\section{NUTRITIONAL ASSESSMENT OF ROBUSTA COFFEE (Coffea canephora) BEANS OF DIFFERENT ORIGINS PROCESSED IN MEXICO}

\author{
Gómez-Merino, F.C. ${ }^{1}$; Trejo-Téllez, L.I. ${ }^{1 *}$; Morales-Ramos, V. ${ }^{1}$; Marín-Garza, T. ${ }^{2}$; Crosby-Galván, M.M. ${ }^{1}$ \\ ${ }^{1}$ Colegio de Postgraduados Campus Montecillo. Carretera México-Texcoco km 36.5. Montecillo, \\ Texcoco, Estado de México. C. P. 56230. ²Universidad Veracruzana. Facultad de Ciencias Biológi- \\ cas y Agropecuarias Zona Córdoba-Orizaba. Carretera Peñuela-Amatlán km 1. Peñuela, Amatlán \\ de los Reyes, Veracruz. C. P. 94945.
}

*Autor de correspondencia: tlibia@colpos.mx

\section{RESUMEN}

El valor nutricional del café como principal bebida en el mundo puede variar de acuerdo a diferentes factores, incluyendo la especie y el origen geográfico donde se produce el grano. En este estudio se evaluaron indicadores de materia orgánica, cenizas, proteínas, grasas y fibra de muestras de café de la variedad Robusta (Coffea canephora) provenientes de México, Brasil y Vietnam. El mayor porcentaje de materia orgánica (96.2) y de grasas (6.7) se observó en muestras de granos provenientes de México. El porcentaje de fibra (21.9) fue mayor en las muestras de Vietnam, en tanto que el de cenizas (4.10) y el de proteinas (10.3) fueron mayores en las muestras de Brasil. Esto comprueba que el origen geográfico de los granos de café influye en su valor nutricional y que en el mercado de México podemos encontrar variación en estos indicadores.

Palabras clave: Coffea, países productores, calidad nutricional, materia orgánica, cenizas, proteínas, grasas, fibras

\section{ABSTRACT}

The nutritional value of coffee as primary beverage in the world can vary according to different factors, including the species and the geographical origin where the beans are produced. In this study, indicators of humidity, organic matter, ash, protein, fat and fiber from coffee samples of the variety Robusta (Coffea canephora) originally from Mexico, Brazil and Vietnam, were evaluated. The highest percentage of humidity (6.1), organic matter (96.2) and fat (6.7) were observed in samples of coffee beans from Mexico. The percentage of fiber (21.9) was higher in the samples from Vietnam, while ash (4.10) and the protein (10.3) were higher in samples from Brazil. This shows that the countries of origin of coffee beans influence their nutritional value and that in the market in Mexico we can find variation in these indicators.

Keywords: Coffea, producing countries, nutritional value, moisture, organic matter, ash, protein, fat, fiber.

Agroproductividad: Vol. 11, Núm. 4, abril. 2018. pp: 25-29.

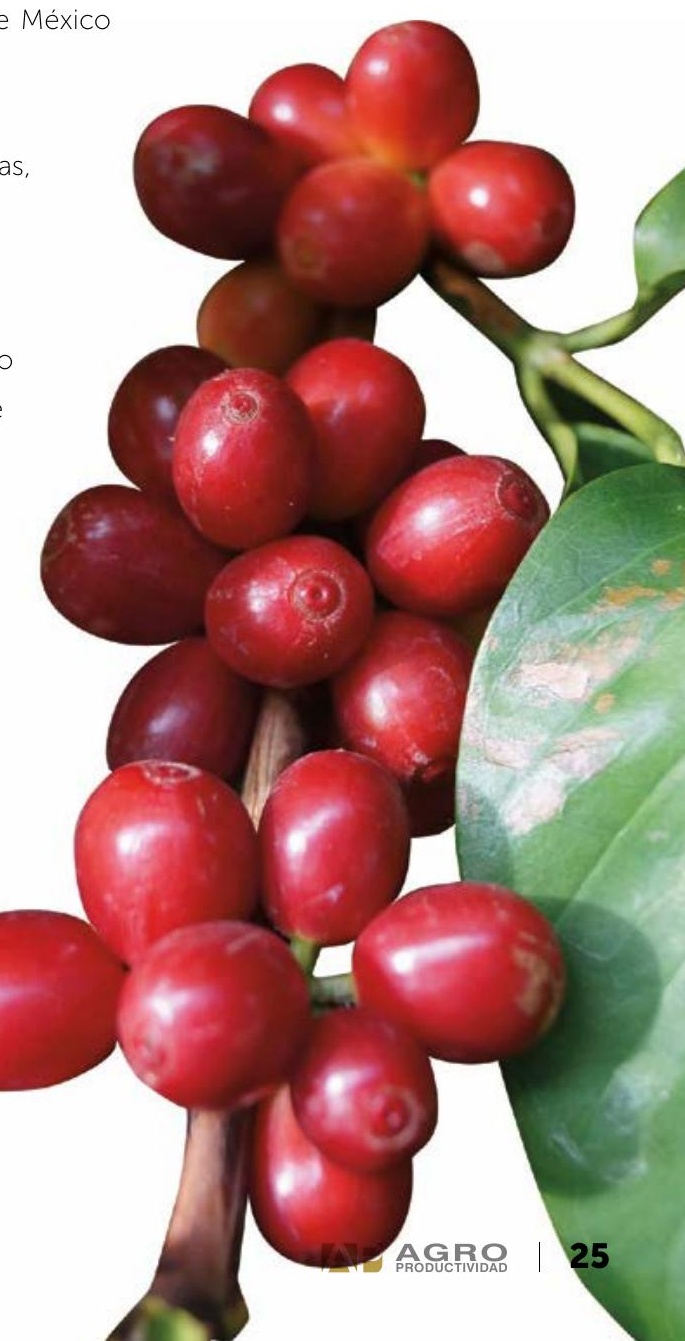




\section{INTRODUCCIÓN}

El café

pertenece a la familia Rubiaceae, y al género Coffea, el cual tiene unas 25 especies. Del total de estas especies, las de mayor cultivo e importancia económica son Coffea arabica (Arabica) y Coffea canephora (Robusta) (ICO, 2017) (Figura 1).
De estos países, sobresalen Brasil, Vietnam, Colombia, Indonesia, Perú, India, Uganda, Honduras, Etiopía y México como los 10 principales productores del aromático en el mundo, y México se consolida como el líder mundial en la producción de cafés orgánicos (ANMECAFE, 2017).

El café ofrece el ingrediente principal de una de las bebidas más populares y apreciadas en el mercado internacional, gracias a su sabor y propiedades estimulantes y refrescantes, y entre las especies se distinguen algunas diferencias como la acidez de las variedades Arabicas y el amargor de las Robustas.

En el mundo el café se produce en la franja tropical en América, África y Asia, principalmente en Angola, Bolivia, Burundi, Camerún, Colombia, Congo, Costa Rica, Costa de Marfil, Cuba, Ecuador, El Salvador, Etiopía, Gabón, Gana, Guatemala, Filipinas, Honduras, India, Indonesia, Kenia, Liberia, Madagascar, Malawi, México, Nicaragua,

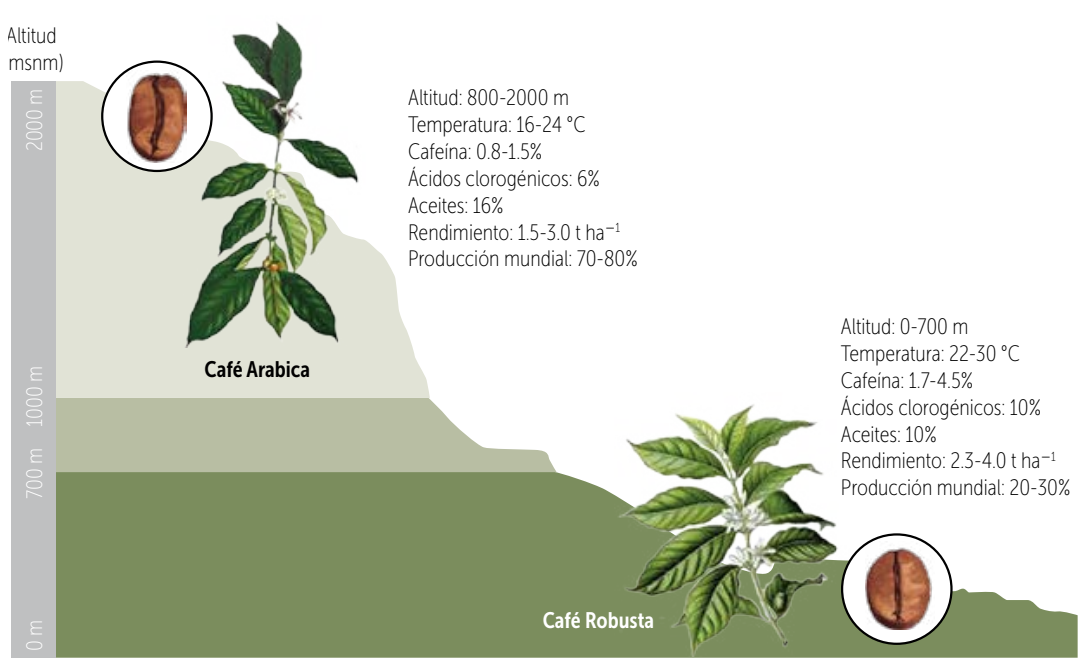

Figura 1. Las dos especies comerciales de café más importantes en el mundo: Coffea arabica (café Arabica) y Coffea canephora (café Robusta). En la figura se describen algunas de las principales características distintivas de cada una de ellas
Panamá, Papúa Nueva Guinea, Paraguay, Perú, República Centroafricana, Ruanda, Sierra Leona, Tanzania, Tailandia, Timor-Leste, Togo, Uganda, Vietnam, Yemen, Zambia y Zimbabue (ICO, 2016) (Figura 2). En 2016, en estos países se produjeron más de 25 millones de quintales de café Arabica y cerca de 14 millones de quintales de café Robusta (ICO, 2017).

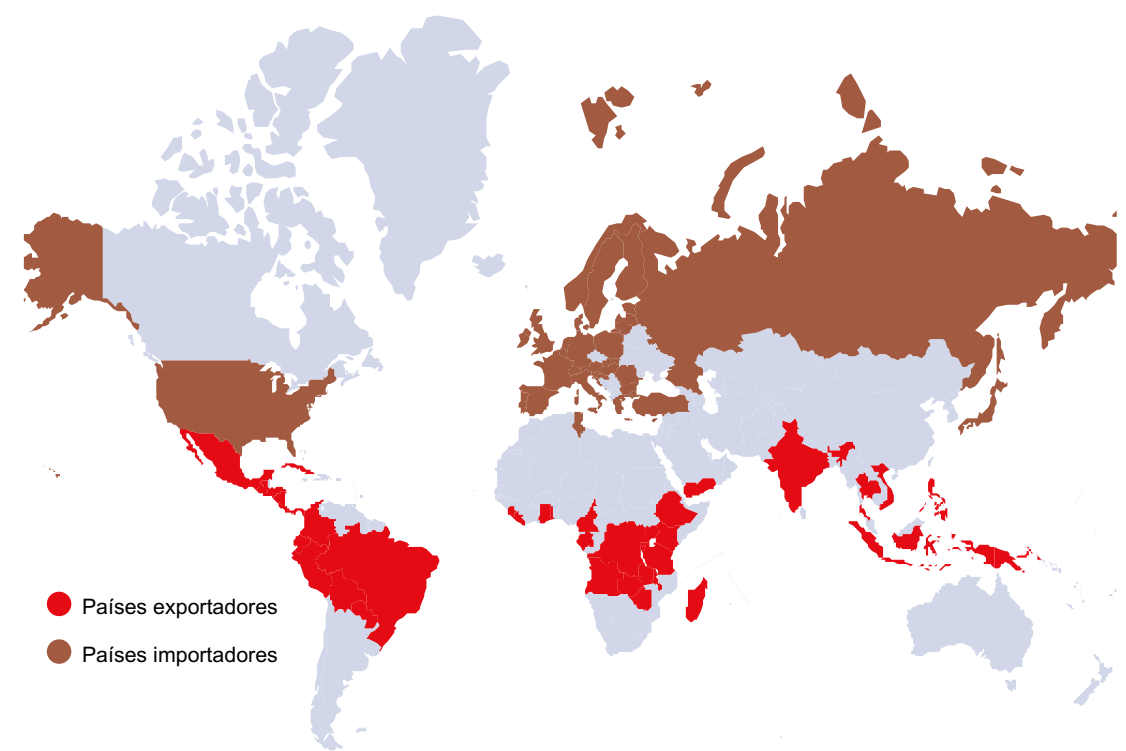

Figura 2. Principales países productores y exportadores (en rojo) e importadores (en marrón) de café en el mundo.
Los granos de café de las variedades de Coffea arabica contienen una mayor concentración de lípidos (un promedio de 14 a 17\% en base seca) que C. canephora, mientras que en C. canephora hay mayor concentración de carbohidratos (42\%), cafeína (2.4\%), ácidos clorogénicos $(3.8 \%)$, ácidos orgánicos $(2.6 \%)$ y cenizas $(4.7 \%)$ que C. arabica (Oestreich-Janzen, 2013), Io que confiere diferente sabor y consistencia a la taza de café. No obstante, el sabor no solo depende de la especie, sino también del origen geográfico de las mismas y del procesamiento a que es sometido el grano, incluyendo el lavado vía húmeda (Puerta-Quintero, 2008; ICO, 2017). En este estudio se evaluó el valor nutricional de granos de café de la variedad Robusta (Coffea canephora) de muestras provenientes de tres paises productores distintos: México, Brasil y Vietnam, específicamente respecto a los contenidos de materia orgánica, cenizas, proteínas, grasas y fibra del grano de café.

\section{MATERIALES Y MÉTODOS}

Muestras de granos de café de la variedad 
Robusta (Coffea canephora) provenientes México, de Brasil y de Vietnam se procesaron siguiendo básicamente la metodología de la AOAC (2015), con algunas adaptaciones.

Peso de biomasa fresca y seca del grano. El peso de la biomasa fresca de los granos se obtuvo al ser pesados por separado en una balanza analítica Pro AV213C (Adventurer Ohaus; Parsippany, NJ, EEUU). Posteriormente los granos fueron secados en una estufa de circulación de aire forzado HCF-125D (Riossa; Monterrey, México) a $70{ }^{\circ} \mathrm{C}$ por $48 \mathrm{~h}$, con lo que se obtuvo el peso de la biomasa seca.

Una vez secos y para hacer las siguientes determinaciones, los granos fueron triturados en un molino de laboratorio tipo Wiley con malla de 1.0 mm (Wiley Modelo 4; Filadelfia, PA, EEUU).

Materia orgánica, cenizas, grasas, proteínas y fibra. Estas determinaciones se hicieron con base en los protocolos descritos por AOAC (2015). Para la determinación de cenizas se usó el protocolo AOAC 942.05, y por diferencia se estimó el porcentaje de materia orgánica. Para la cuantificación de grasas se usó el protocolo AOAC 920.39 y para el de proteínas el AOAC 2001.11. La determinación de fibras se hizo con base en el protocolo AOAC 985.29 .

\section{Análisis estadístico de datos}

Con los datos obtenidos se realizó un análisis de varianza para un diseño experimental completamente azar y cuando se detectaron diferencias estadísticas, se realizó la comparación de medias por el método de Tukey $(P \leq 0.05)$ con el paquete estadístico SAS.

\section{RESULTADOS Y DISCUSIÓN}

Los contenidos porcentuales de las variables medidas para determinar la calidad nutricional de los granos de café Robusta (Coffea canephora) de diferentes orígenes que se procesaron y fueron analizados en este estudio aparecen en el Cuadro 1.

De acuerdo con el NFI (2009), los valores de humedad en café tostado pueden oscilar entre 5.4 y $6.2 \%$. Sin embargo, estos valores no fueron determinados en las muestras aquí analizadas.

Los contenidos de materia orgánica fueron mayores en las muestras de café mexicano, seguidas de las de Vietnam y por último las de Brasil. No obstante, los valores aquí encontrados están por arriba del rango reportado por el NFI (2009) y Oestreich-Janzen (2013), los cuales son inferiores al 95\%.

De acuerdo con el NFI (2009) y Oestreich-Janzen (2013), el contenido de cenizas en granos de café oscila entre 3.9 y $4.7 \%$. En este estudio, los valores de esta variable oscilaron entre 3.8 y $4.2 \%$, lo cual puede considerarse dentro de los valores ya reportados. El mayor contenido de cenizas se encontró en los granos de café de Brasil.

Los contenidos de proteínas en granos de café oscilan del 13 al 15\% (NFI, 2009), por lo que los valores reportados en el presente estudio son inferiores a este rango. Entre nuestras determinaciones, los valores más altos los mostraron los granos de Brasil (10.3), y los más bajos los de Vietnam (9.8), con valores intermedios para los granos de México (10.1).

\begin{tabular}{|c|c|c|c|c|c|}
\hline \multirow{2}{*}{$\begin{array}{c}\text { País de origen } \\
\text { del grano }\end{array}$} & Materia orgánica & Cenizas & Proteínas & Grasas & Fibra \\
\hline & \multicolumn{5}{|c|}{$\%$ (en base seca) } \\
\hline México & $96.2 a$ & $3.8 \mathrm{c}$ & $10.1 b$ & $6.7 a$ & $17.8 \mathrm{c}$ \\
\hline Brasil & $95.8 \mathrm{c}$ & $4.2 \mathrm{a}$ & $10.3 a$ & $5.4 c$ & $19.8 b$ \\
\hline Vietnam & $96.0 \mathrm{~b}$ & $4.0 \mathrm{~b}$ & $9.8 c$ & $6.1 b$ & $21.9 a$ \\
\hline
\end{tabular}

Medias con letra distinta en la misma columna son estadisticamente diferentes de acuerdo a la prueba de (Tukey, $P \leq 0$.05). Cada valor es el promedio de tres repeticiones.
De acuerdo con el NFI (2009), los contenidos de grasas en granos de café pueden variar entre 15 y $16 \%$, en tanto que Oestreich-Janzen (2013) reporta valores del 11 a 17\%. En general, todas las muestras analizadas en este trabajo tuvieron contenidos de grasas muy inferiores a los reportados en otros estudios. De estos valores, las muestras de México tuvieron las medias más altas (6.7), y las de Brasil las más bajas (5.4); el café de Vietnam tuvo valores medios respecto a esta variable (6.1).

El contenido de fibras en granos de café es de aproximadamente 20\% (NFI, 2009). En nuestro estudio, las 
muestras analizadas estuvieron muy cercanas a este valor, con la media más alta en muestras de Vietnam (21.9) y la más baja en muestras de México (17.8).

Con excepción de los valores de proteínas y grasas, las demás variables analizadas en este estudio (materia orgánica, cenizas y fibras) mostraron similitud con otros reportes.

Además del origen geográfico y el procesamiento de tostado del café, así como el tiempo al que es sometido, es necesario puntualizar que muchos compuestos químicos reaccionan e interactúan en todas las etapas del procesamiento del café para obtener un producto final con una gran diversidad y complejidad de estructuras.

\section{Variación en la composición química de acuerdo a la especie}

Está bien documentado que los perfiles de compuestos químicos varía entre especies de café (i.e. Coffea canephora vs. C. arabica). A fin de tener un comparativo entre estas dos especies, en el Cuadro 2 se muestra resultados de diferentes investigaciones respecto a la composición de granos.

De acuerdo a Puerta-Quintero (2011), el café Robusta tostado presenta contenidos de humedad promedio de $4.76 \%$, en tanto que en el café Arabica este contenido es mayor, y puede variar desde el 5 hasta el 11\% (Mazzafera, 1999; Food-Info, 1999; NFI, 2009).

De acuerdo con Meléndez-Márquez (2006), el porcentaje de materia orgánica en café Robusta es de aproximadamente $93 \%$, en tanto que el café Arabica es cercano al $90 \%$.

En relación al porcentaje de cenizas en café Robusta se reportan valores de 3.6 a 4.7\% (Food-Info, 1999; Mon-
tero-Rodríguez et al., 1999; Meléndez-Márquez, 2006; Puerta-Quintero, 2011; Oestreich-Janzen, 2013). Para café Arabica los reportes señalan que están dentro del rango de 3.5 a 4.6\% (Montero-Rodríguez et al., 1999; Meléndez-Márquez, 2006; Oestreich-Janzen, 2013; PuertaQuintero, 2011).

En café Robusta se reportan contenidos de proteínas del 12 al 16\% (Meléndez-Márquez, 2006; Temis-Pérez et al., 2011; Puerta-Quintero, 2011), en tanto que en Arabica estos contenidos oscilan entre 12 y 15\% (Montero-Rodríguez et al., 1999; Meléndez-Márquez, 2006; Temis-Pérez et al., 2011).

De acuerdo con Food-Info (1999), en café Arabica el contenido promedio de carbohidratos es de $57 \%$, mientras que en café Robusta es de $61 \%$.

Los porcentajes de azúcares libres (glucosa, fructosa y sacarosa) en café Robusta pueden variar desde un 3.5 a un 7.5\% (Meléndez-Márquez, 2006; Oestreich-Janzen, 2013), mientras que en Arabica oscila entre 5 y 9\% (Meléndez-Márquez, 2006; Redgwell y Fischer, 2006).

Respecto a los contenidos de grasas o lípidos en granos de café Robusta, éstos pueden variar entre 10 y $15 \%$ en base seca (Food-Info, 1999; Montero-Rodríguez et al., 1999; Temis-Pérez et al., 2011; Puerta-Quintero, 2011; Meléndez-Márquez, 2006; Oestreich-Janzen, 2013). En café Arabica, estos valores pueden ser más altos, en general por arriba de 15\% en base seca (Food-Info, 1999; Mazzafera, 1999; Montero-Rodríguez et al., 1999; Meléndez-Márquez, 2006; NFI, 2009; Puerta-Quintero, 2011; Oestreich-Janzen, 2013).

En cuanto a fibras, en café Robusta se han reportado valores promedio de 17 a 20\% (Meléndez-Márquez, 2006; Puerta-Quintero, 2011), mientras que en Arabica el valor es de aproximadamente 16\% (Sánchez y Anzola, 2012).

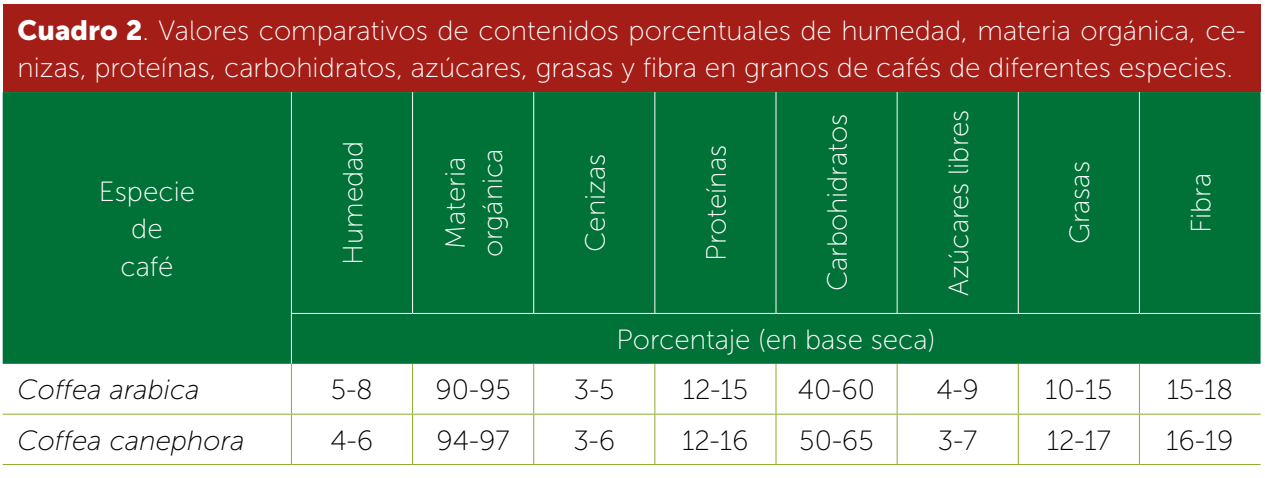

Variación en la composición química de acuerdo al origen En el Cuadro 3 se muestran resultados promedio de algunos valores porcentuales de cafés Robusta y Arabicas de diferentes orígenes geográficos.

Los rangos de los valores de las variables reportadas para Brasil, 
Uganda, Kenia y Colombia se basan en las revisiones de Puerta-Quintero (2011), y los Etiopía y Colombia fueron reportados por Sánchez y Anzola (2012). Se puede apreciar que el origen geográfico de los granos influye en la composición química de éstos. En general, los cafés colombianos presentan los valores más altos en las variables medidas.

\section{CONCLUSIÓN}

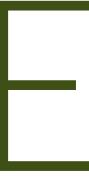

$n$ este estudio se pudo comprobar que el origen geográfico de los granos de café influye en su composición nutricional. En general, materia orgánica y grasas fueron mayores en los granos provenientes de México, en tanto que los granos de Brasil mostraron medias más altas para cenizas y proteínas. El contenido porcentual de fibras fue mayor en granos de Vietnam. Para futuros estudios comparativos, habría que estandarizar las metodologías de determinación de estos constituyentes.

\section{LITERATURA CITADA}

AMECAFE. 2017. Datos sobre café en México. Asociación Mexcicana de la Cadena Productiva del Café. https://amecafe.org.mx

AOAC. 2015. Official Methods of Analysis of AOAC International. Food composition, additives and natural contaminants. Gaithersburg, MA, USA.

Food-Info.1999. Química del café: Universidad de Wageningen Wageningen, Paises Bajos. http://www.food-info.net/es/ products/coffee/chemistry.htm

ICO. 2016. ICO Annual Meeting. Annual review 2014-2015 Strengthening the global coffee sector through international cooperation. International Coffee Organization. London, UK.

ICO 2017. Seminars and Workshops. International Coffee Organization. http://www.ico.org/workshop.asp

Mazzafera P. 1999. Chemical composition of defective coffee beans Food Chemistry 64: 547-554
Meléndez-Márquez H.A. 2006. Establecimiento y validación de curvas de calibración NIRS para café oro de Honduras. Tesis. Universidad Zamorano. Francisco Morazán, Honduras. 37 p.

Montero-Rodríguez M.G., Talón-Marques M.A., Sánchez-Muñiz F.J. 1999. Consumo de café y colesterol sérico. Grasas y Aceites 50: $141-152$

NFI. 2009. Coffee bean, roasted, ground. National Food Institute Technical University of Denmark. http://www.foodcomp.dk/ v7/fcdb_details.asp?Foodld $=0103$

Oestreich-Janzen S. 2013. Chemistry of coffee. In: Mande L., Ben H.W. (eds.). Comprehensive Natural Products. Elsevier, Oxford. pp. 1-26. doi: 10.1016/B978-008045382-8.00708-5

Puerta-Quintero G.I. 2008. Riesgos para la calidad por la comercialización de café pergamino húmedo. Avances Técnicos Cenicafé 373: 1-4

Puerta-Quintero G.I. 2011. Composicion quimica de una taza de café Avances Técnicos Cenicafé 414: 1-12.

Redgwell R., Fischer M. 2006. Coffee carbohydrates. Braz. J. Plant Physiol. 18: 165-174.

Sánchez A.D., Anzola V.C. 2012. Chemical characterization of the coffee silverskin (Coffea arabica) in varieties Colombia and Caturra. Rev. Colomb. Quim. 41: 211-226.

Temis-Pérez A.L., López-Malo A., Sosa-Morales M.E. 2011. Producción de café (Coffea arabica L.): cultivo, beneficio, plagas y enfermedades. Temas Selectos de Ingeniería de Alimentos 5: 54-74. 